\title{
Differential expression of fatty acid desaturase 1 in triple negative breast cancer.
}

Shahan Mamoor, MS ${ }^{1}$

1'shahanmamoor@gmail.com

East Islip, NY 11730

Women diagnosed with triple negative breast cancer can benefit neither from endocrine therapy nor from HER2-targeted therapies ${ }^{1}$. We mined published microarray datasets ${ }^{2,3}$ to determine in an unbiased fashion and at the systems level genes most differentially expressed in the primary tumors of patients with breast cancer. We report here significant differential expression of the gene encoding fatty acid desaturase 1, FADS1, when comparing the tumor cells of patients with triple negative breast cancer to normal mammary ductal cells ${ }^{2}$. FADS1 was also differentially expressed in bulk tumor in human breast cancer ${ }^{3}$. FADS1 mRNA was present at significantly increased quantities in TNBC tumor cells relative to normal mammary ductal cells. Analysis of human survival data revealed that expression of FADS1 in primary tumors of the breast was correlated with recurrence-free survival in patients with basal-like and normal-like subtype cancer, while within triple negative breast cancer, primary tumor expression of FADS1 was correlated with distant metastasis-free survival in patients with basal-like 1 and immunomodulatory subtype disease. FADS1 may be of relevance to initiation, maintenance or progression of triple negative breast cancers. We previously reported ${ }^{4}$ that the fatty acid desaturase 2, FADS2, was also among the genes most differentially expressed in triple negative and in early-onset breast cancers ${ }^{2,3}$ in humans. Together, the data suggest that these enzymes ${ }^{5}$, their transcriptome-wide differential expression, marked transcriptional up-regulation and accessible catalytic sites may make them suitable for therapeutic targeting.

Keywords: breast cancer, FADS1, fatty acid desaturase 1, systems biology of breast cancer, targeted therapeutics in breast cancer. 
Patients with hormone receptor positive disease can benefit from endocrine therapy ${ }^{6}$ : aromatase inhibition such as that provided by exemestane or letrozole, or estrogen receptor antagonism such as that provided by tamoxifen or fulvestrant. Patients whose tumors overexpress the human epidermal growth factor 2 (HER2) can be treated with HER2-targeted therapies: HER2 inhibitors, immunoglobulin ${ }^{7}$ - or small molecule-based ${ }^{8}$. As triple negative breast cancer (TNBC) is defined by its lack of HER2 expression as well as by its lack of expression of the hormone receptor estrogen, patients diagnosed with triple negative breast cancer will not benefit from either of these treatment strategies.

Understanding the transcriptional composition of triple negative breast cancers in humans can facilitate development of novel therapeutics and contribute to efforts to prevent progression to metastatic stages. We mined published microarray data ${ }^{2,3}$ to understand at the transcriptome level and in an unbiased fashion genes most differentially expressed in the tumor cells of patients with triple negative breast cancer.

\section{Methods}

We utilized datasets GSE38959 ${ }^{2}$ and GSE109169³ for this differential gene expression analysis of female breast cancer in conjunction with GEO2R. GSE38959 was generated using Agilent-014850 Whole Human Genome Microarray 4x44K G4112F technology with $n=13$ samples of normal mammary gland ductal cells and $n=30$ samples of tumor cells from patients with triple negative breast cancer; analysis was performed using platform GPL4133. GSE109169 was generated using Affymetrix Human Exon 1.0 ST Array technology with $n=25$ normal breast tissue and $n=25$ tumors of the breast; analysis was performed using platform GPL5175. The tissues from this dataset are paired tissues ( 25 tumors matching 25 normal breast tissues from 25 patients). The Benjamini and Hochberg method of $p$-value adjustment was used for ranking of differential expression but raw $p$-values were used to assess statistical significance of global differential expression. Log-transformation of data was auto-detected, and the NCBI-generated category of platform annotation was used. A statistical test was performed to evaluate whether FADS1 expression was significantly different between primary breast tumors and breast tissue using a two-tailed t-test.

For Kaplan-Meier survival analysis, we used the Kaplan-Meier plotter tool ${ }^{9}$ for correlation of FADS1 mRNA expression levels with recurrence-free survival (RFS) in $n=630$ patients with basal subtype cancer, $n=998$ patients with luminal A subtype cancer, $n=673$ patients with luminal B subtype cancer, $n=401$ patients with HER $2+$ cancer, and $n=63$ patients with normal-like subtype cancer. In patients with triple negative breast cancer ${ }^{7}$, we analyzed distant metastasis-free survival (DMFS) for $n=171$ patients with basal-like 1 subtype TNBC, $n=71$ patients with basal-like 2 subtype TNBC, $n=219$ patients with immunomodulatory subtype TNBC, $n=138$ patients with luminal androgen receptor subtype TNBC, $n=130$ patients with mesenchymal subtype TNBC, and $n=39$ patients with mesenchymal stem-like subtype TNBC.

\section{Results}

We performed discovery of genes associated with triple negative breast cancer in humans by mining two independently published microarray datasets ${ }^{2,3}$.

\section{FADS1 is differentially expressed in primary tumors and tumor cells of the breast.}

Comparison of the tumor cells of patients with triple negative breast cancer to normal mammary ductal cells ${ }^{2}$ revealed fatty acid desaturase 1 , encoded by FADS1, as among the genes whose expression was most significantly different in human TNBC as compared to the normal breast (Chart 1$)$. When sorting each of the genes expressed in the tumor cells of patients with triple negative breast cancer based on significance of difference as compared to normal mammary ductal cells, FADS1 ranked 67 out of 45015 total transcripts, equating to $99.9 \%$ differential expression (Chart 1). Differential expression of FADS1 in the tumor cells of patients with triple negative breast cancer was statistically significant (Chart $1 ; p=2.14 \mathrm{E}-13)$. 
Analysis of a second microarray datase ${ }^{3}$, here comparing 25 normal breast tissues to 25 tumors of the breast revealed that FADS1 was also among the genes most differentially expressed in human breast cancer, here in the tumors of women diagnosed with early-onset breast cancer (Chart 2). When sorting each of the genes expressed in tumors of the breast based on significance of difference as compared to normal breast tissue, FADS1 ranked 8581 out of 19076 total transcripts, equating to $55.0 \%$ differential expression (Chart 2). Differential expression of FADS1 in female breast cancer was statistically significant (Chart 2; $p=2.59 \mathrm{E}-02)$.

These data indicated that differential expression of FADS1 was not an artifact of a single microarray dataset, was not an artifact of FACS isolation, and suggested that perturbed expression of FADS1 was likely a transcriptional feature of triple negative breast cancers and early-onset human breast cancers.

FADS1 is expressed at significantly higher levels in triple negative tumor cells as compared to normal cells of the breast.

We obtained exact mRNA expression levels for FADS1 - from normal mammary ductal cells of the breast and from tumor cells of patients with triple negative breast cancer - to understand the magnitude and direction of FADS1 expression change in TNBC. FADS1 mRNA transcript was present at higher levels in triple negative tumor cells of the breast as compared to normal mammary ductal cells (Figure 1). Increased expression of FADS1 in primary tumor-isolated triple negative breast cancer cells was statistically significant (Figure 1: $p<0.0001$ ). FADS1 was expressed at $45.5 \pm 38.5$ arbitrary units (A.U.) in normal mammary ductal cells, while it was expressed at $346.5 \pm 214$ A.U. in tumor cells of patients with triple negative breast cancer. We calculated a mean fold change of 7.61 when comparing triple negative breast cancer cells to normal mammary ductal cells.

FADS1 expression correlates with survival outcomes in basal-like and normal-like human breast cancer.

We performed Kaplan-Meier survival analysis to evaluate potential relationships between primary tumor FADS1 mRNA expression levels and survival outcomes in patients with breast cancer, when analyzing outcomes based on the molecular subtype of the patient (PAM50 classification): luminal A, luminal B, HER2+, normal-like, and basal-like. We observed a correlation between FADS1 expression and recurrence-free survival (RFS) in patients with basal-like subtype breast cancer (Figure 2; log rank $p$-value: 0.068 for recurrence-free survival, hazard ratio: $0.76(0.57-1.02)$ (Fig. 2)). FADS1 mRNA levels were a positive prognostic indicator in basal-like breast cancer patients. Median RFS was 29.23 months for basal-like patients with low tumor expression of FADS1 while median RFS was 55.66 months for basal-like patients with high tumor expression of FADS1 (Chart 3).

We also observed a statistically significant correlation between FADS1 expression and recurrence-free survival (RFS) in patients with normal-like subtype breast cancer (Figure 2; log rank $p$-value: 0.0098 for recurrence-free survival, hazard ratio: 3.93 (1.28-12.06) (Fig. 2)). FADS1 mRNA levels were a negative prognostic indicator in normal-like breast cancer patients. Median RFS was, on average, markedly greater for normal-like patients with low tumor expression of FADS 1 as compared to normal-like patients with high tumor expression of FADS1 (Fig. 2).

FADS1 primary tumor expression was not correlated with recurrence-free survival in luminal A (Figure 2; log rank $p$-value: 0.17 for RFS, hazard ratio: 1.25 (0.9-1.74) (Fig. 2), luminal B subtype (Figure 2; $\log$ rank $p$-value: 0.11 for RFS, hazard ratio: 1.27 (0.95-1.71) (Fig. 2)), or in HER2+ breast cancer (Figure 2; log rank $p$-value: 0.3 for RFS, hazard ratio: 0.83 (0.58-1.18) (Fig. 2)). 


\section{References}

1. Foulkes, W.D., Smith, I.E. and Reis-Filho, J.S., 2010. Triple-negative breast cancer. New England journal of medicine, 363(20), pp.1938-1948.

2. Komatsu, M., Yoshimaru, T., Matsuo, T., Kiyotani, K., Miyoshi, Y., Tanahashi, T., Rokutan, K., Yamaguchi, R., Saito, A., Imoto, S. and Miyano, S., 2013. Molecular features of triple negative breast cancer cells by genome-wide gene expression profiling analysis. International journal of oncology, 42(2), pp.478-506.

3. Chang, J.W., Kuo, W.H., Lin, C.M., Chen, W.L., Chan, S.H., Chiu, M.F., Chang, I.S., Jiang, S.S., Tsai, F.Y., Chen, C.H. and Huang, P.H., 2018. Wild-type p53 upregulates an early onset breast cancer-associated

4. Mamoor, S., 2021. Differential expression of CDC28 protein kinase regulatory subunit 1B in triple negative breast cancer.

5. Guillou, H., Zadravec, D., Martin, P.G. and Jacobsson, A., 2010. The key roles of elongases and desaturases in mammalian fatty acid metabolism: Insights from transgenic mice. Progress in lipid research, 49(2), pp.186-199.

6. Spring, L.M., Gupta, A., Reynolds, K.L., Gadd, M.A., Ellisen, L.W., Isakoff, S.J., Moy, B. and Bardia, A., 2016. Neoadjuvant endocrine therapy for estrogen receptor-positive breast cancer: a systematic review and meta-analysis. JAMA oncology, 2(11), pp.1477-1486.

7. Slamon, D., Eiermann, W., Robert, N., Pienkowski, T., Martin, M., Press, M., Mackey, J., Glaspy, J., Chan, A., Pawlicki, M. and Pinter, T., 2011. Adjuvant trastuzumab in HER2-positive breast cancer. New England journal of medicine, 365(14), pp.1273-1283.

8. Park, J.W., Liu, M.C., Yee, D., Yau, C., van’t Veer, L.J., Symmans, W.F., Paoloni, M., Perlmutter, J., Hylton, N.M., Hogarth, M. and DeMichele, A., 2016. Adaptive randomization of neratinib in early breast cancer. New England Journal of Medicine, 375(1), pp.11-22.

9. Györffy, B., Lanczky, A., Eklund, A.C., Denkert, C., Budczies, J., Li, Q. and Szallasi, Z., 2010. An online survival analysis tool to rapidly assess the effect of 22,277 genes on breast cancer prognosis using microarray data of 1,809 patients. Breast cancer research and treatment, 123(3), pp.725-731.

10. Lehmann, B.D., Bauer, J.A., Chen, X., Sanders, M.E., Chakravarthy, A.B., Shyr, Y. and Pietenpol, J.A., 2011. Identification of human triple-negative breast cancer subtypes and preclinical models for selection of targeted therapies. The Journal of clinical investigation, 121(7), pp.2750-2767. 


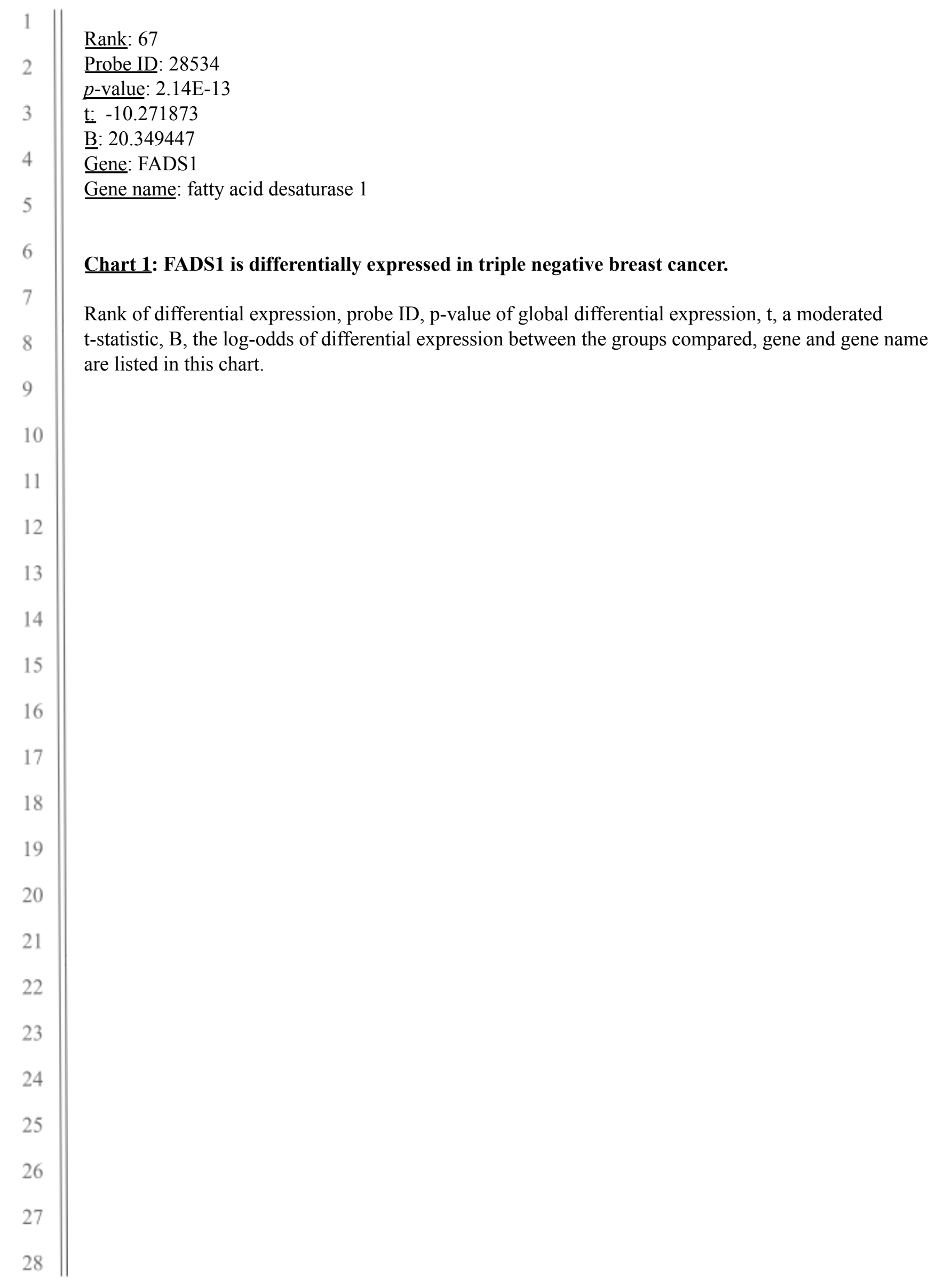




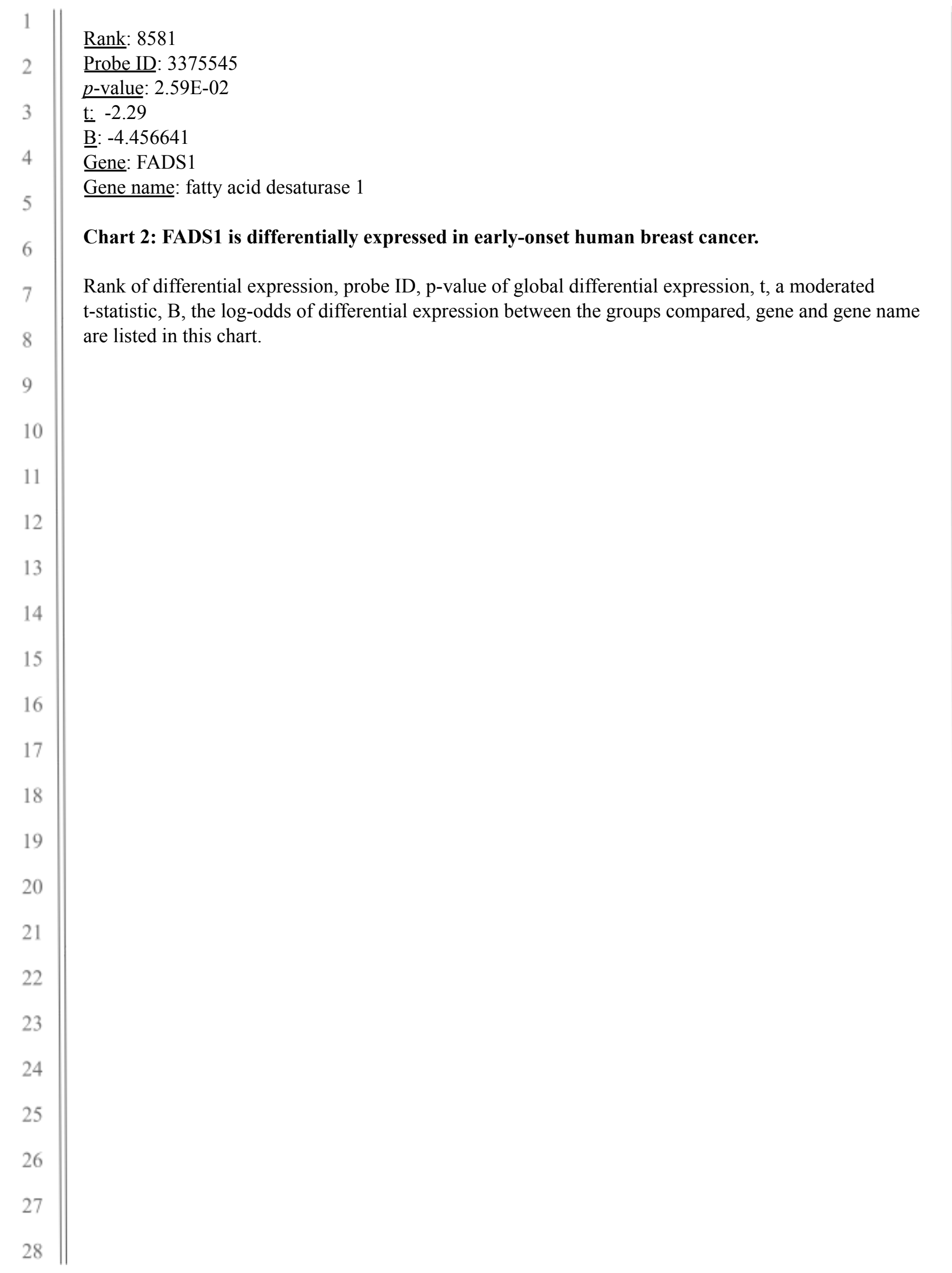


FADS1

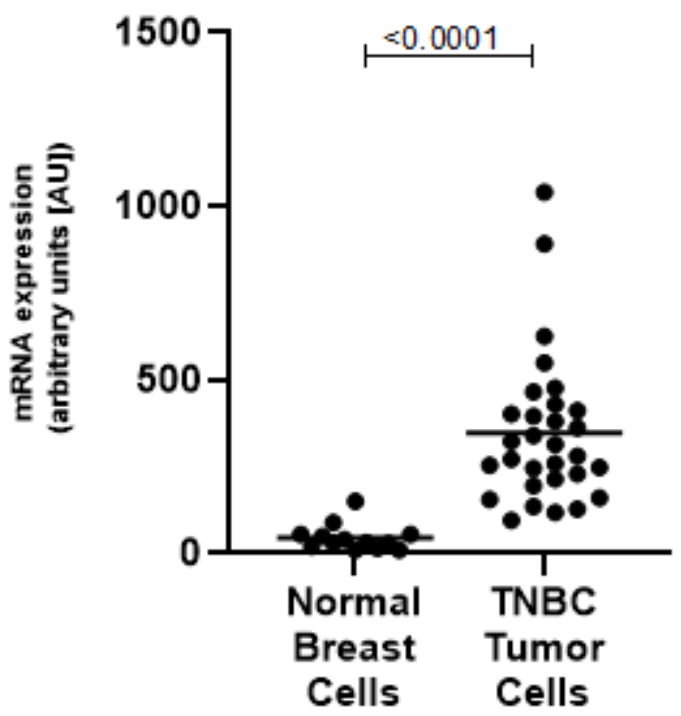

Figure 1: FADS1 is expressed at significantly higher levels in primary TNBC tumor cells as compared to normal cells of the breast.

The mRNA expression level of FADS1 in normal mammary ductal cells (left) and in the isolated primary tumor cells of patients with triple negative breast cancer (right) is graphically depicted with the result of a statistical test evaluating significance of difference in FADS1 expression between normal cells of the breast and TNBC tumor cells, a $p$-value, listed above. 


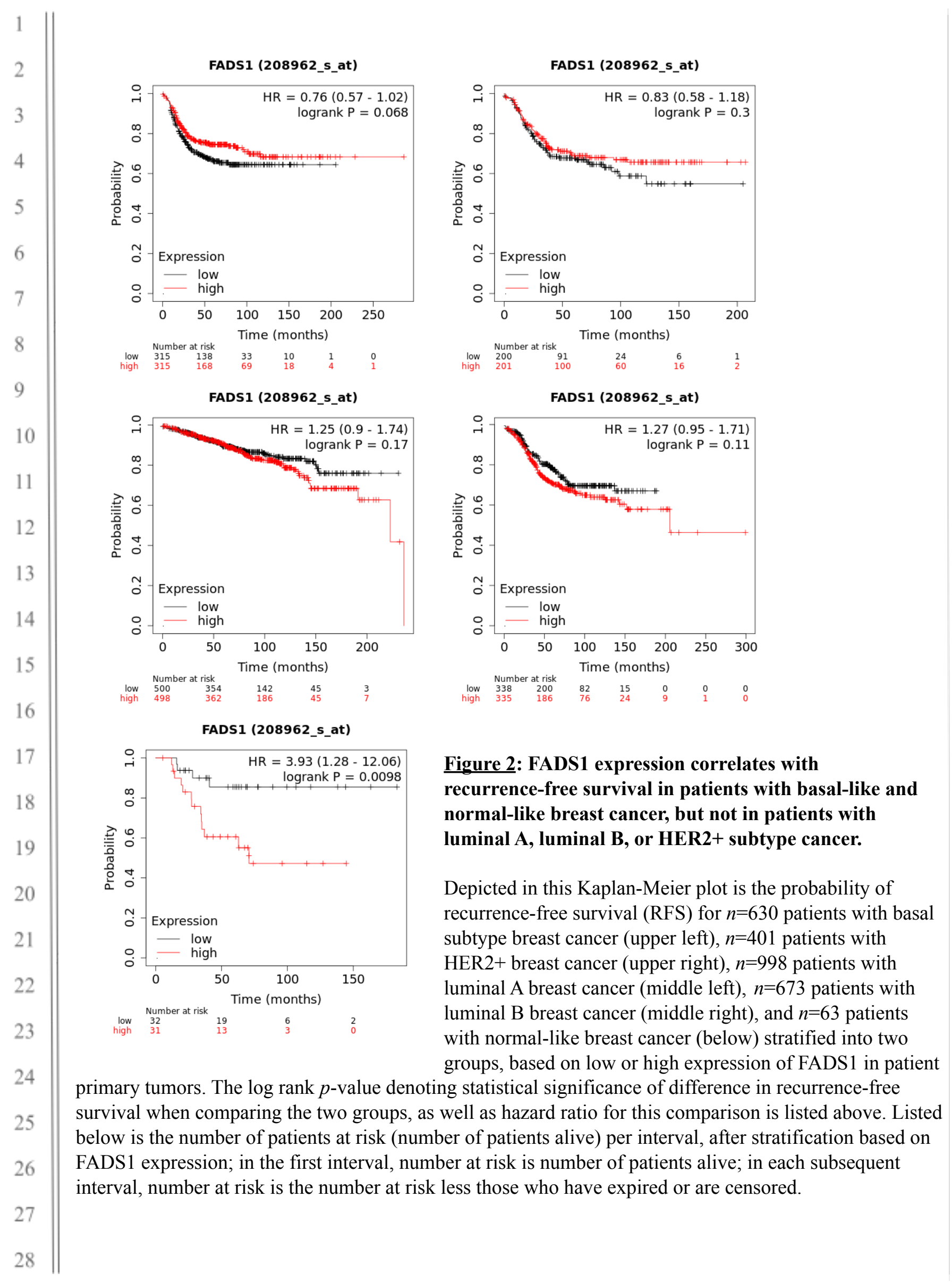




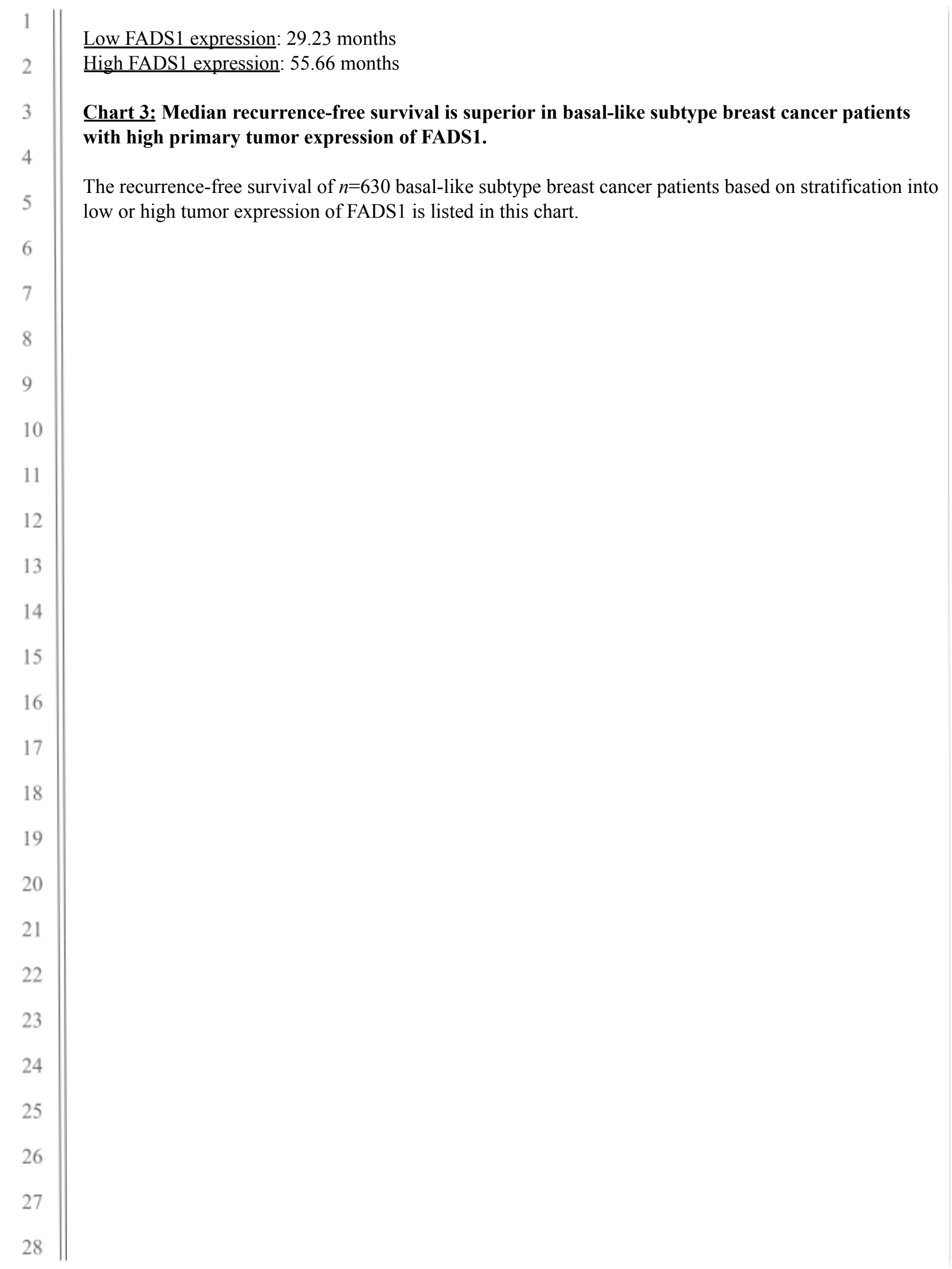



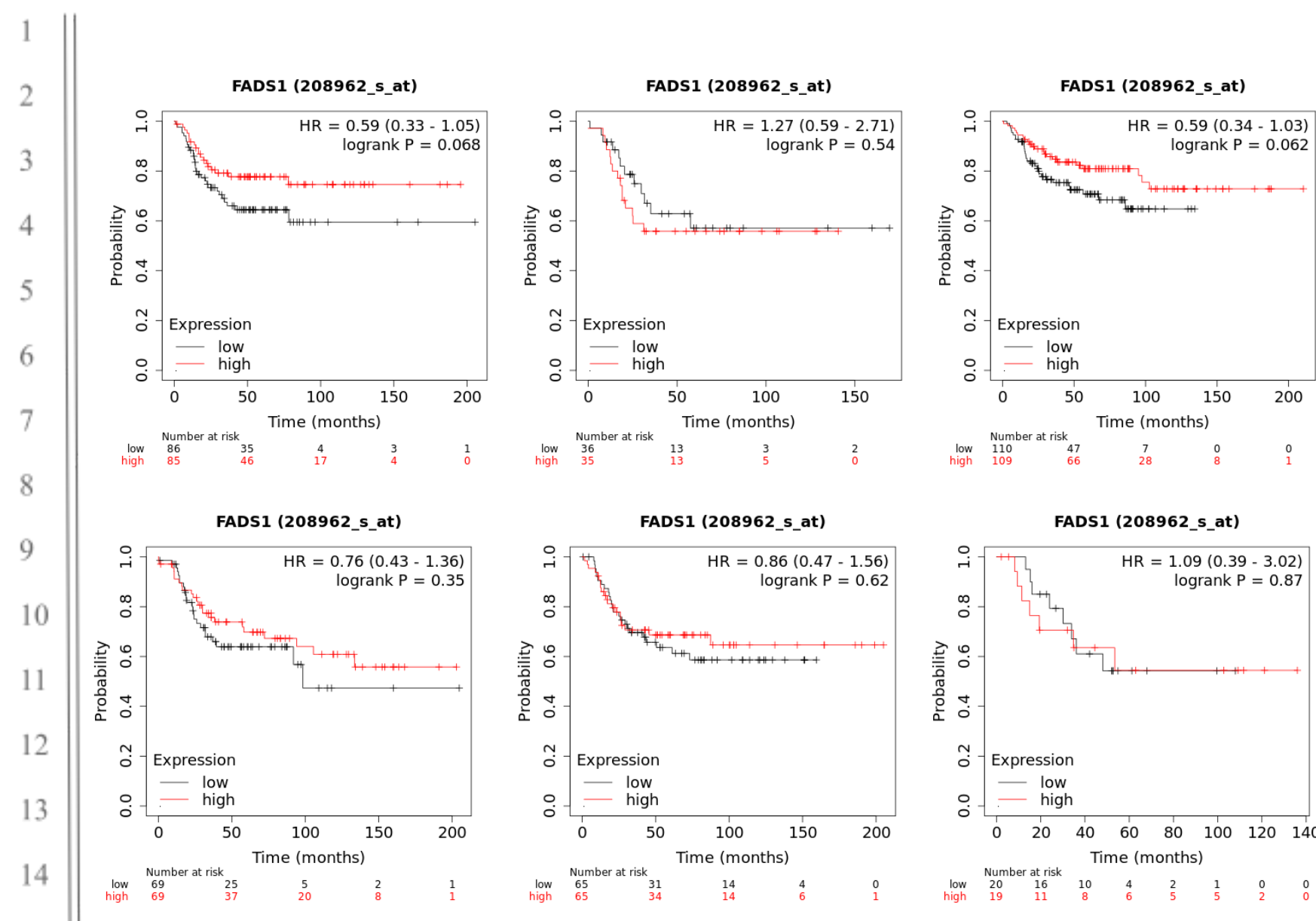

Figure 3: FADS1 expression correlates with distant metastasis-free survival in triple negative breast cancer patients whose tumors are of the basal-like 1 and immunomodulatory molecular subtypes.

Depicted in this Kaplan-Meier plot is the probability of distant metastasis-free survival (DMFS) for $n=171$ patients with basal-like 1 subtype TNBC (upper left), $n=71$ patients with basal-like 2 subtype TNBC (upper right), $n=219$ patients with immunomodulatory subtype TNBC (middle left), $n=138$ patients with luminal androgen receptor subtype TNBC (middle right), $n=130$ patients with mesenchymal subtype TNBC (lower left), and $n=39$ patients with mesenchymal stem-like subtype TNBC (lower right), stratified into two groups, based on low or high expression of FADS1 in the primary tumors of patients with TNBC. The log rank p-value denoting statistical significance of difference in distant metastasis-free survival when comparing the two groups, as well as hazard ratio for this comparison is listed above. Listed below is the number of patients at risk (number of patients alive) per interval, after stratification based on FADS1 expression; in the first interval, number at risk is number of patients alive; in each subsequent interval, number at risk is the number at risk less those who have expired or are censored. 


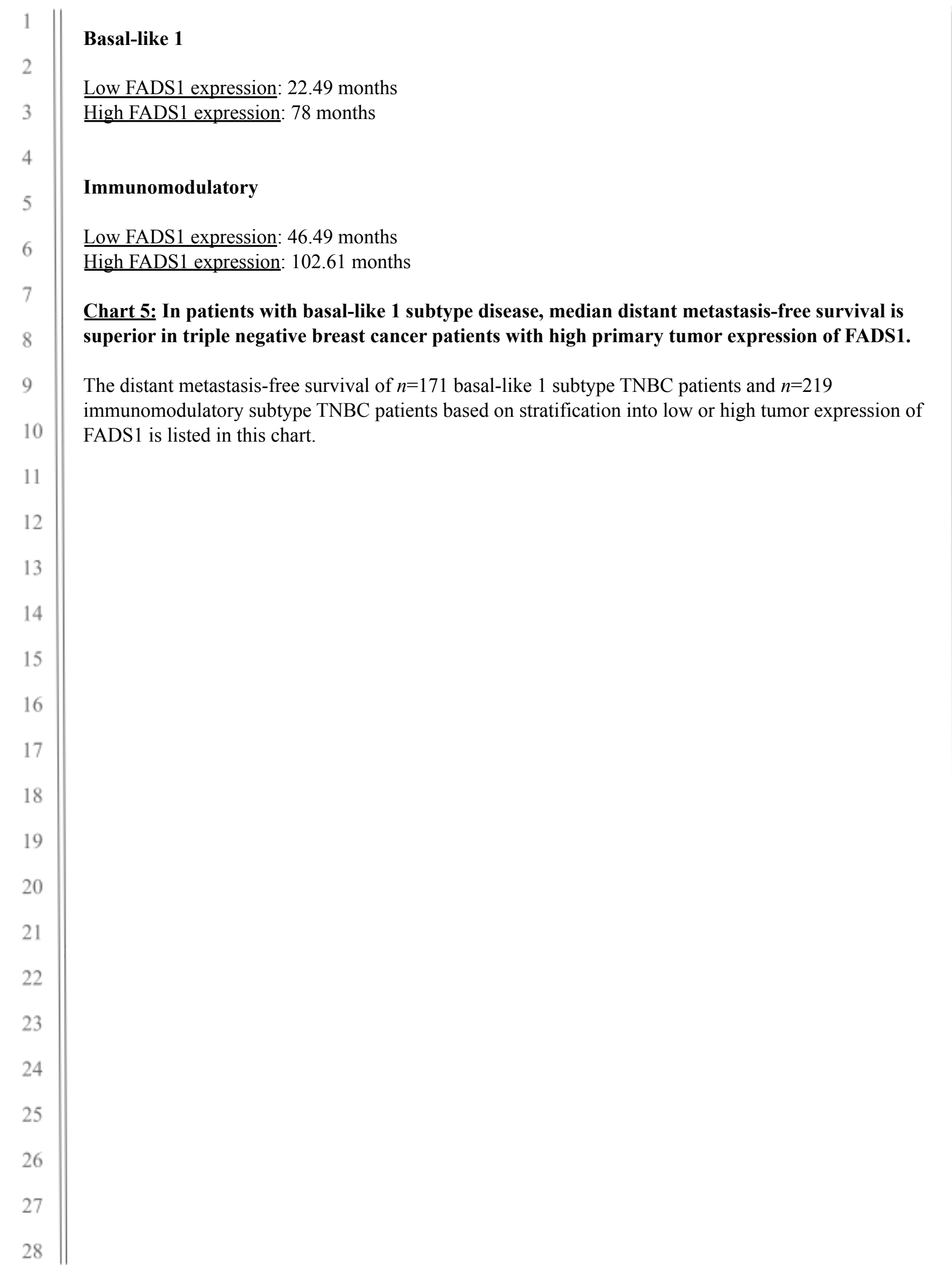

